\title{
Experimental realization and characterization of an electronic Lieb lattice
}

\author{
Marlou R. Slot ${ }^{1}$, Thomas S. Gardenier ${ }^{1}$, Peter H. Jacobse', Guido C. P. van Miert ${ }^{2}$, Sander N. Kempkes ${ }^{2}$, \\ Stephan J. M. Zevenhuizen ${ }^{1}$, Cristiane Morais Smith ${ }^{2}$, Daniel Vanmaekelbergh' ${ }^{1}$ and Ingmar Swart ${ }^{1 \star}$
}

\begin{abstract}
Geometry, whether on the atomic or nanoscale, is a key factor for the electronic band structure of materials. Some specific geometries give rise to novel and potentially useful electronic bands. For example, a honeycomb lattice leads to Dirac-type bands where the charge carriers behave as massless particles ${ }^{1}$. Theoretical predictions are triggering the exploration of novel two-dimensional (2D) geometries ${ }^{2-10}$, such as graphynes and the kagomé and Lieb lattices. The Lieb lattice is the 2D analogue of the 3D lattice exhibited by perovskites ${ }^{2}$; it is a square-depleted lattice, which is characterized by a band structure featuring Dirac cones intersected by a flat band. Whereas photonic and cold-atom Lieb lattices have been demonstrated ${ }^{11-17}$, an electronic equivalent in 2D is difficult to realize in an existing material. Here, we report an electronic Lieb lattice formed by the surface state electrons of $\mathrm{Cu}(111)$ confined by an array of carbon monoxide molecules positioned with a scanning tunnelling microscope. Using scanning tunnelling microscopy, spectroscopy and wavefunction mapping, we confirm the predicted characteristic electronic structure of the Lieb lattice. The experimental findings are corroborated by muffin-tin and tight-binding calculations. At higher energies, second-order electronic patterns are observed, which are equivalent to a super-Lieb lattice.
\end{abstract}

The Lieb lattice is a square-depleted lattice, described by three sites in a square unit cell, as illustrated in Fig. 1a. Two of the sites (indicated in red) are neighboured by two other sites. The third site in the unit cell (blue) has four neighbours. In the remainder of this article, these sites will be referred to as edge (red) and corner (blue) sites, respectively. This geometry results in an electronic band structure exhibiting two characteristic features: two dispersive bands, which form a Dirac cone at the $\mathrm{M}$ point in the first Brillouin zone, and a flat band crossing the Dirac point (Fig. 1b). It is well established that Dirac cones give rise to unusual behaviour, such as effectively massless fermions. Similarly, flat bands may potentially facilitate the realization of magnetic order ${ }^{18,19}$, give rise to the fractional quantum (spin) Hall and the quantum anomalous Hall effect ${ }^{20,21}$, and enhance the critical temperature of superconductors ${ }^{22,23}$.

The electronic band structure of the Lieb lattice can be calculated from the following tight-binding Hamiltonian:

$$
\mathcal{H}=\sum_{i} \epsilon_{i} c_{i}^{\dagger} c_{i}-t \sum_{\langle i, j\rangle}\left(c_{i}^{\dagger} c_{j}+\text { H.c. }\right)-t^{\prime} \sum_{\langle\langle i, j\rangle\rangle}\left(c_{i}^{\dagger} c_{j}+\text { H.c. }\right)
$$

where $\epsilon_{i}$ and $t\left(t^{\prime}\right)$ indicate the on-site energy of site $i$ and the (next-)nearest-neighbour hopping constants, respectively. Taking only nearest-neighbour hopping into account and using the same on-site energy for the three sites results in the band structure shown in Fig. 1b. The flat band exclusively contains electronic states which are localized on edge sites. In contrast, all sites contribute to the dispersing bands converging to the Dirac cone. Hence, the local density of states (LDOS) exhibits a characteristic spatial variation, see Fig. 1c.

Thus far, a 2D electronic Lieb lattice has not been realized. In principle, lithography could be used to impose a Lieb pattern on a $2 \mathrm{D}$ electron gas ${ }^{7,24}$. Alternatively, a strategy similar to the one employed for generating artificial graphene could be used-that is, assembling a molecular lattice on a substrate featuring a surface state to force the electrons into the desired geometry ${ }^{25}$. In the following, we will choose the latter approach and describe how atomic scale manipulation of carbon monoxide molecules on $\mathrm{Cu}(111)$ with a scanning tunnelling microscope is used to generate and characterize an electronic Lieb lattice.

The design of the molecular Lieb lattice is not trivial for several reasons. First, the Lieb lattice has four-fold rotational symmetry, whereas substrates that exhibit a surface state close to the Fermi energy such as $\mathrm{Cu}(111)$ have hexagonal symmetry. Furthermore, the carbon monoxide $(\mathrm{CO})$ molecules on $\mathrm{Cu}(111)$ act as repulsive scatterers, confining the electrons to the space between the $\mathrm{CO}$ molecules ${ }^{25-28}$. This implies that the CO molecules should compose the anti-lattice of the electronic Lieb lattice. Our design consists of a CO square lattice, which defines the trivial anti-lattice of a square lattice, with one $\mathrm{CO}$ placed in the centre of four $\mathrm{CO}$ molecules to form the anti-lattice of a depleted square lattice (see Fig. 1d). This design was recently proposed independently by Qiu and colleagues ${ }^{29}$. The size of the unit cell is chosen to be $6 \sqrt{3} a_{0} \times 10 a_{0}(\approx 2.66 \mathrm{~nm} \times 2.56 \mathrm{~nm})$, where $a_{0}=0.256 \mathrm{~nm}$ is the $\mathrm{Cu}(111)$ nearest-neighbour distance. Two factors play a critical role in the design. First, this arrangement of CO molecules provides the best approximation to the perfect four-fold symmetry of the Lieb lattice on the hexagonal $\mathrm{Cu}(111)$ substrate. Furthermore, the size of the unit cell determines the position of the bands of the lattice with respect to the Fermi level of the $\mathrm{Cu}(111)^{25}$. With the lattice constants described above, the flat band is close to the Fermi level (see below).

To establish whether the design described above confines the electrons into an electronic Lieb lattice, we performed calculations based on the nearly-free electron model, in which the CO molecules are modelled by a muffin-tin potential. The band structure calculated using this approach is given by the black curve in Fig. 1e. These results can be reproduced well using a tight-binding model including orbital overlap and next-nearest-neighbour interactions $\left(t^{\prime} / t=0.6\right)$, see the grey curve in Fig. 1e. Hence, the arrangement of

${ }^{1}$ Debye Institute for Nanomaterials Science, Utrecht University, PO Box 80 000, 3508 TA Utrecht, the Netherlands. ${ }^{2}$ Institute for Theoretical Physics, Utrecht University, PO Box 80 089, 3508 TB Utrecht, the Netherlands. *e-mail: i.swart@uu.nl 
a

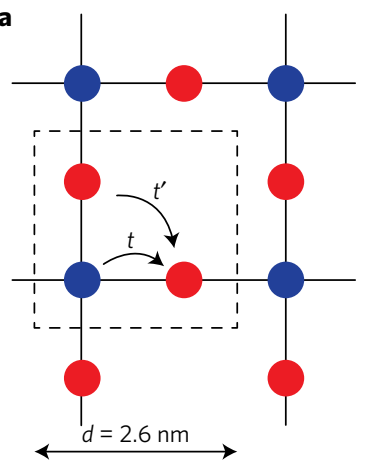

b

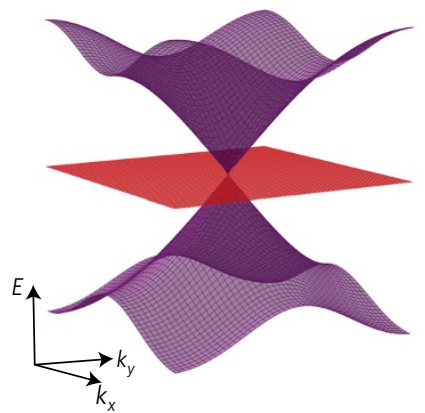

c

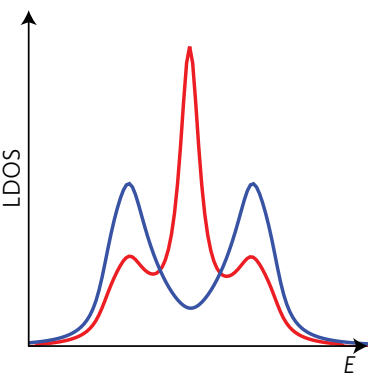

d

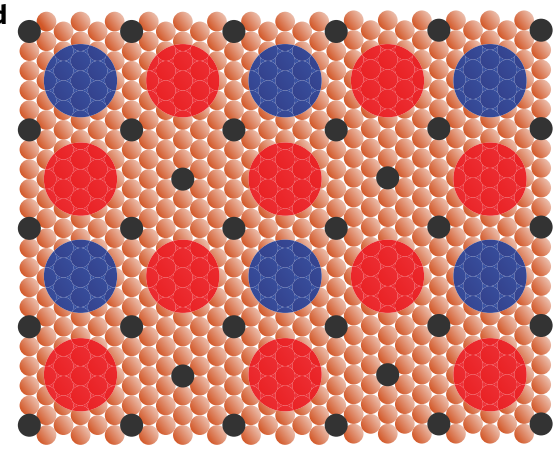

e

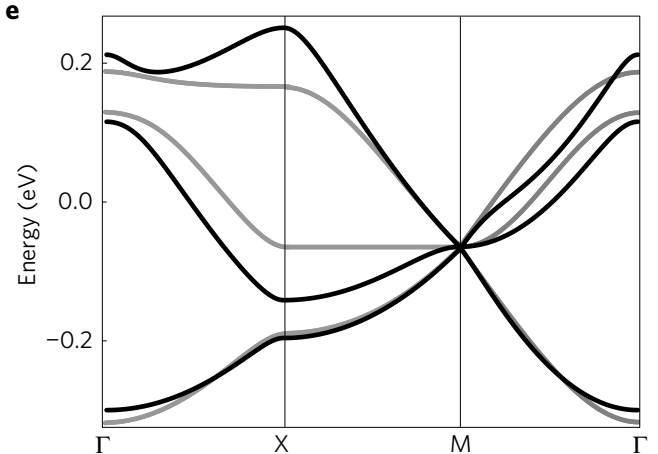

Figure 1 | Designing an electronic Lieb lattice. a, Geometric structure of the Lieb lattice. The unit cell (black dashed line) contains two edge sites and one corner site, indicated in red and blue, respectively. $\mathbf{b}$, Band structure of the Lieb lattice around the M point of the Brillouin zone, taking into account only nearest-neighbour hopping. c, Calculated local density of states at edge (red) and corner (blue) sites. d, Geometric arrangement of CO molecules (black) on a $\mathrm{Cu}(111)$ surface to generate an electronic Lieb lattice. Red and blue circles correspond to the edge and corner sites in a. e, Band structure from muffin-tin (black) calculations along the high-symmetry lines of the Brillouin zone, overlaid with the tight-binding result using parameters that provide the best agreement with the muffin-tin simulations (grey).

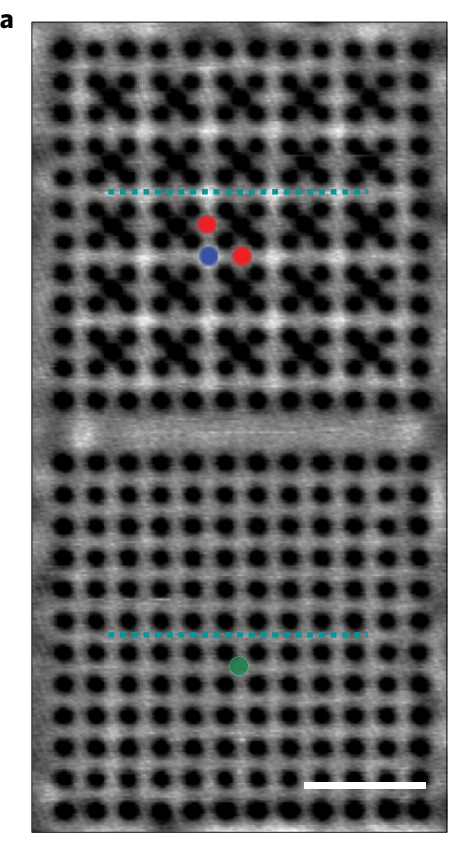

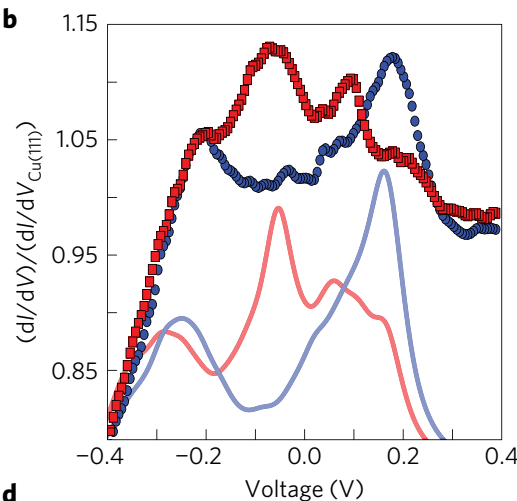

d

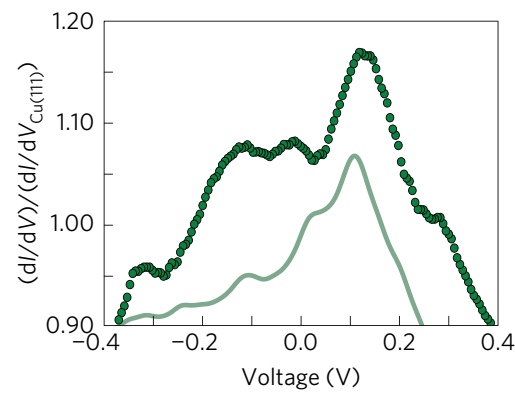

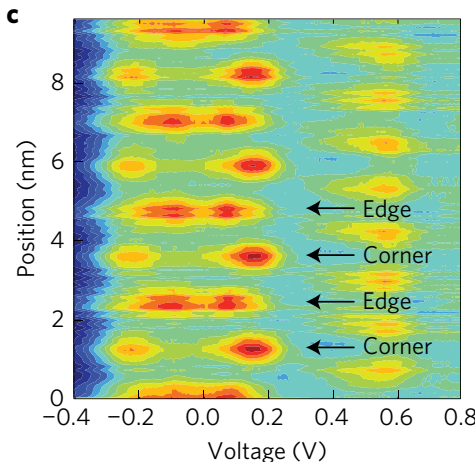

e

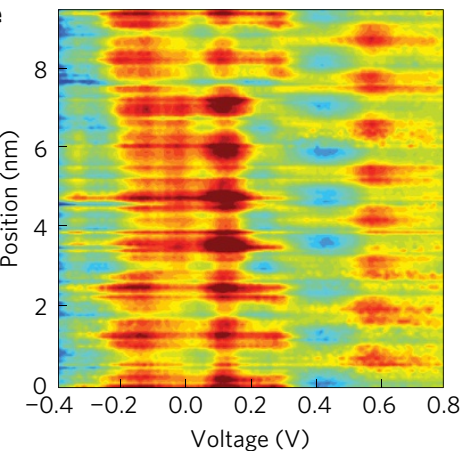

Figure 2 | Electronic structure of a Lieb lattice. a, Image of a $5 \times 5$ Lieb (top) and square (bottom) lattice. Two edge sites and one corner site of the Lieb lattice are indicated in red and blue, respectively. The green circle indicates a site of the square lattice. Imaging parameters: $V=50 \mathrm{mV}, I=1 \mathrm{nA}$. Scale bar, $5 \mathrm{~nm}$. b, Normalized differential conductance spectra acquired above edge (red squares) and corner (blue circles) sites and local density of states at these sites calculated using the tight-binding method (solid lines). c, Contour plot of 100 normalized spectra taken along the dashed line indicated in a. The features observed in the spectra shown in $\mathbf{b}$ can be clearly recognized (see arrows). d,e, Same as $\mathbf{b}, \mathbf{c}$, but for a square lattice. Note that the spectrum on the square lattice is qualitatively different from the spectra acquired on the Lieb lattice. 

Lowest-energy band

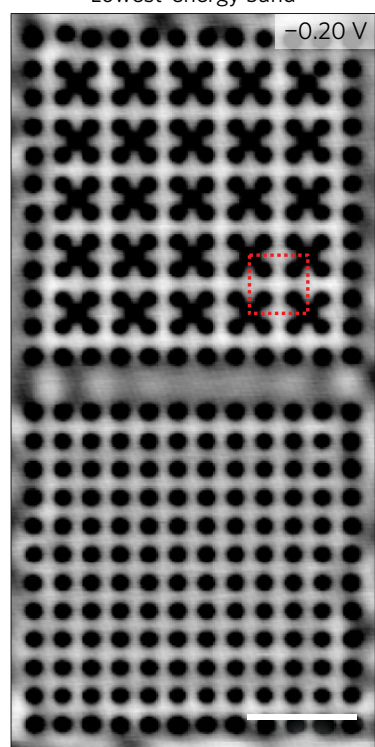

d

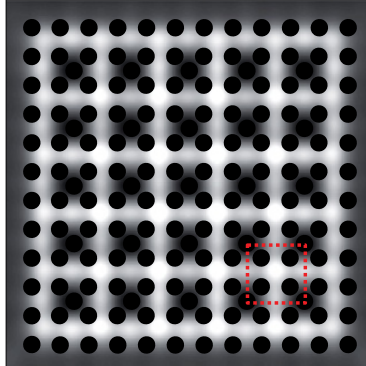

g

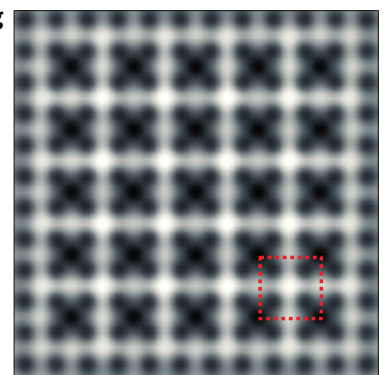

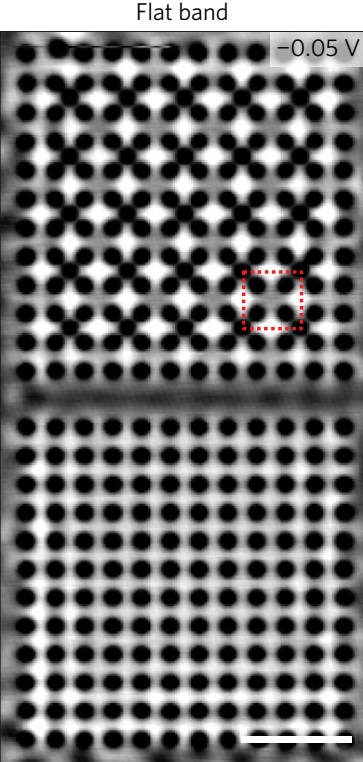

e

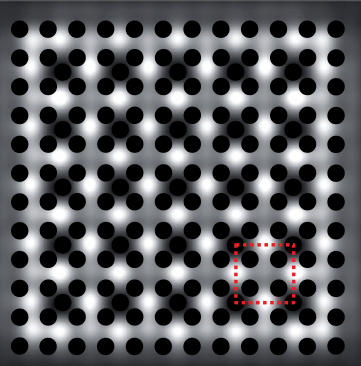

h

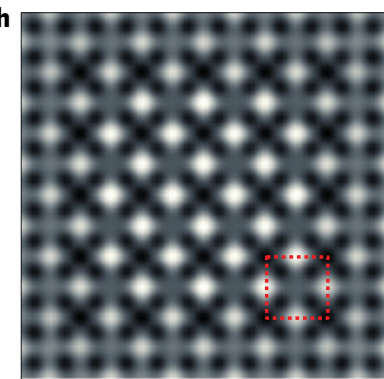

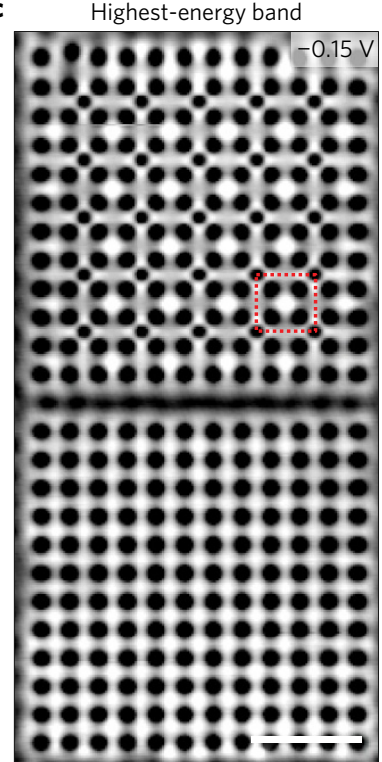
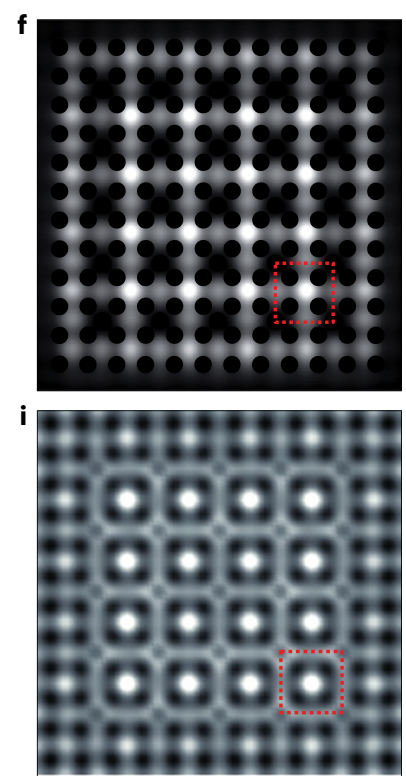

Figure 3 | Wavefunction mapping. a-c, Differential conductance maps acquired above a Lieb (top) and square (bottom) lattice at - $0.20 \mathrm{~V}$, $-0.05 \mathrm{~V}$, and $+0.15 \vee$, respectively. Scale bars, $5 \mathrm{~nm}$. d-f, Differential conductance maps for the Lieb lattice at these energies simulated using tight-binding. Black circles representing the $\mathrm{CO}$ molecules have been added manually to the tight-binding maps. $\mathbf{g}$-i, Same as $\mathbf{d}$-f, but calculated using the muffin-tin model.

$\mathrm{CO}$ molecules on $\mathrm{Cu}(111)$ shown in Fig. 1d generates an electronic Lieb lattice. The large $t^{\prime} / t$ ratio shows that next-nearest-neighbour hopping in this system is important. This can be rationalized by the fact that the distance between individual CO molecules is fairly large on the atomic scale. A detailed description of the correspondence between the nearly-free electron and tight-binding calculations is given in the Supplementary Information.

A lattice of $5 \times 5$ unit cells was assembled in the way shown in Fig. 2a. To provide further evidence that any observed features are due to the Lieb lattice, a square lattice was created immediately next to the Lieb lattice. Differential conductance spectra were acquired above various positions of the lattice (indicated by the blue and red circles in Fig. 2a). The spectra were normalized by the average spectrum acquired on the clean $\mathrm{Cu}(111)$ surface, analogously to ref. 25. The resulting spectra above corner (blue) and edge sites (red) are shown in Fig. 2b. We first focus on the spectrum acquired above a corner site (blue). Two peaks are observed, one at $V=-0.20 \mathrm{~V}$ and one at $+0.18 \mathrm{~V}$. These peaks can be assigned to the lowest- and highest-energy bands in the nearest-neighbour tight-binding model of the Lieb lattice. In between these two peaks, the LDOS reaches a minimum, which should correspond to the Dirac point. In contrast, the edge-site spectrum (red) exhibits a maximum, which is located at $V=-0.07 \mathrm{~V}$. This peak can be assigned to the flat band. The neighbouring peaks are again due to the lowest- and highestenergy bands.

In principle, a flat band should give rise to an (infinitely) narrow feature in the LDOS. In contrast, the peak at $V=-0.07 \mathrm{~V}$ observed above the edge sites is fairly broad. We attribute this broadening to the influence of next-nearest-neighbour hopping, as well as to the limited lifetime of the electrons in the surface state.

The experimentally observed differential conductance spectra are reproduced very well when next-nearest-neighbour hopping is included in tight-binding calculations of a finite lattice (Fig. 2b). Next-nearest-neighbour hopping is essential to account for the observed asymmetry in the LDOS of the low- and high-energy bands (blue spectrum, peaks at $-0.20 \mathrm{~V}$ and $+0.18 \mathrm{~V}$ ), as well as 
a

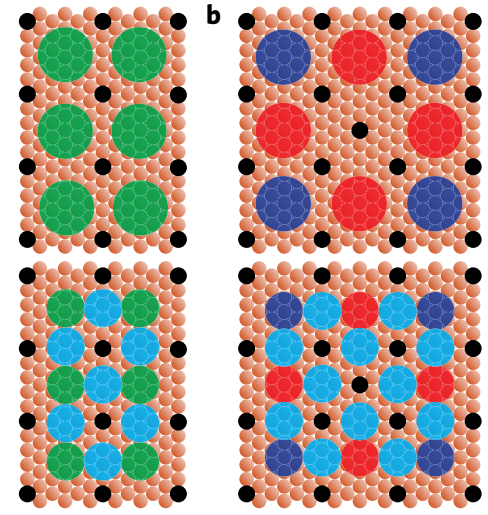

e

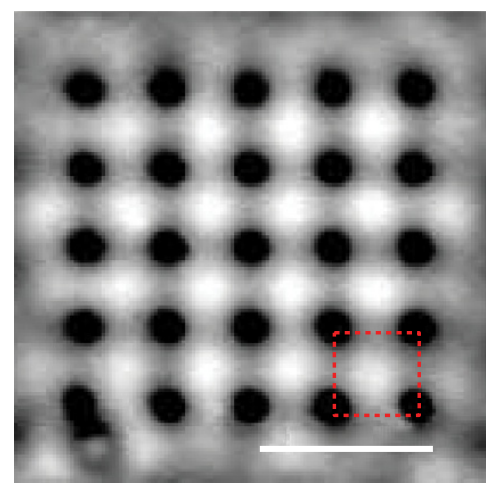

c

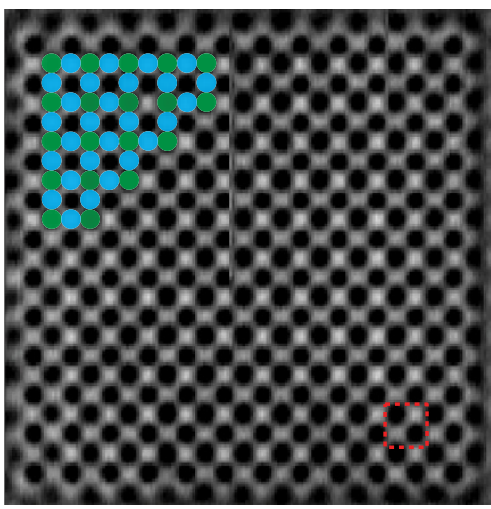

f

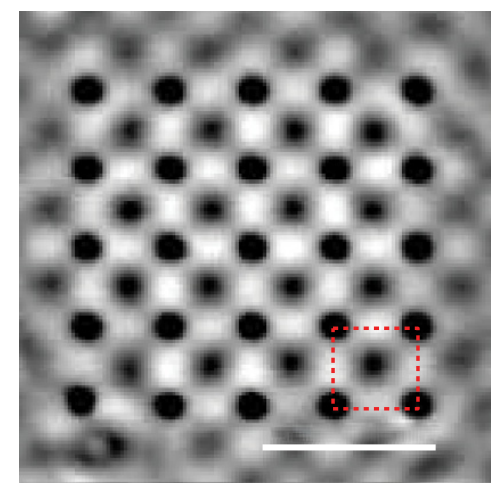

d

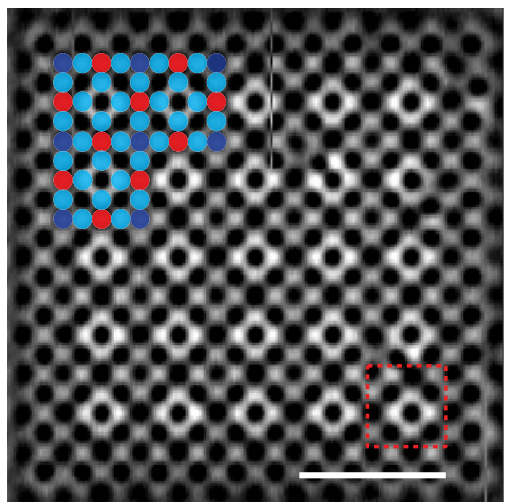

g

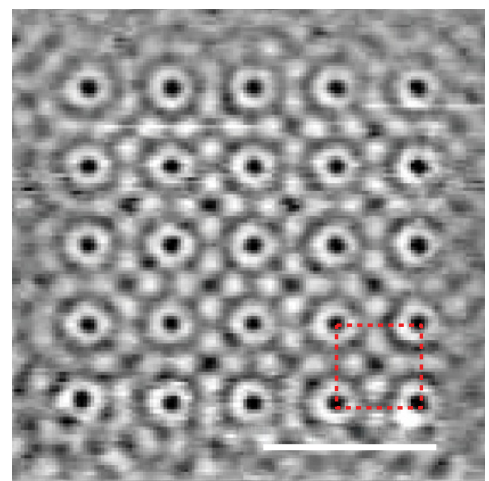

Figure 4 | Higher-order effects. a,b, Schematic picture to show extra sites, resulting in a quasi-Lieb and quasi-super-Lieb lattice, respectively.

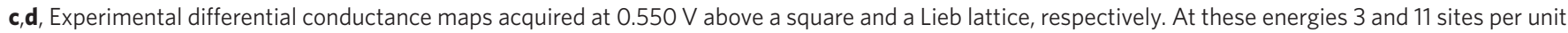
cell are required to provide an adequate description of the wavefunction localization. $\mathbf{e}-\mathbf{g}$, Experimental differential conductance maps acquired above a square lattice at $-0.300 \mathrm{~V},-0.150 \mathrm{~V}$, and $0.575 \mathrm{~V}$, respectively. In each of these maps, the unit cell is indicated by a red dashed line. Note that each unit cell still only contains one $\mathrm{CO}$ molecule. All scale bars denote $5 \mathrm{~nm}$.

for the peak at $0.09 \mathrm{~V}$ in the edge-site spectrum. A fit of the tightbinding result to the experimental data yields $t=(89 \pm 15) \mathrm{meV}$, which is in excellent agreement with earlier results ${ }^{25}$. Using this hopping parameter, we calculate the Fermi velocity of the electrons in the Dirac cones to be $v_{\mathrm{F}}=(3.5 \pm 0.6) \times 10^{5} \mathrm{~m} \mathrm{~s}^{-1}$.

To investigate the spatial distribution of the electronic states, we acquired differential conductance maps (see below), as well as 100 spectra along the line indicated in Fig. 2a. This line starts and ends at an edge site and passes four corner sites. The resulting contour plot is shown in Fig. 2c. The peaks described above can be clearly recognized for each site, demonstrating that the LDOS features are a property of the lattice.

For comparison, a differential conductance spectrum acquired over a site in the square lattice is shown in Fig. 2d, while a contour plot showing 125 spectra along a line is shown in Fig. 2e. The spectra along the line again demonstrate the similarity of the features for equivalent sites (Fig. 2e). Importantly, the spectra are qualitatively different from the spectra obtained over the Lieb lattice and display a good agreement with the LDOS calculated for the square lattice using the tight-binding model (using the same parameters as for the Lieb lattice) (see Fig. 2d). This further demonstrates that the features observed in the differential conductance spectra shown in Fig. $2 b$ are due to the Lieb lattice.

Figure 3 shows several experimental and simulated constantheight differential conductance maps of the two lattices. For the square lattice, all equivalent sites appear identical at all three energies. In contrast, for the Lieb lattice at $V=-0.20 \mathrm{~V}$, both the edge and corner sites contribute significantly to the density of states. At the energy of the flat band $(V=-0.05 \mathrm{~V})$, the contribution of the edge sites to the density of states dominates. At $V=+0.15 \mathrm{~V}$, again both corner and edge sites contribute significantly, with the first being dominant. The simulated maps using the tight-binding model (Fig. 3d-f) and using the muffin-tin approach (Fig. $3 g-i$ ) reproduce the features observed experimentally.

A careful inspection of the contour plots shown in Fig. $2 \mathrm{c}$ and e indicates that for both the square and Lieb lattice there is structure in the spectra at higher energy (around $V=+0.60 \mathrm{~V}$ ). For both lattices, these high-energy states are localized in between different sites. To account for these states in the tight-binding calculations, additional basis functions need to be included. This can be done by adding sites in between the original sites. To first order, the simple square lattice is then described by a three-site quasi-Lieb model, with corner and edge sites having different on-site energies (Fig. 4a). Likewise, the Lieb lattice is described by a super-Lieb (Fig. 4b) geometry involving 11 sites per unit cell. Differential conductance maps of the high-energy states of the square and Lieb lattice with indicated unit cells are shown in Fig. 4c and d, respectively. Note that 3 and 11 sites are required to describe the unit cells, respectively. Using this model, we again simulated differential conductance maps. The experimental and simulated maps at higher energy are in good agreement.

The peak positions with respect to the Fermi energy can be shifted to lower energies by increasing the lattice constant ${ }^{25}$. We make use of this effect to access states with even higher energy in the square lattice. Figure $4 \mathrm{e}-\mathrm{g}$ shows differential conductance maps of a square lattice with a four times larger unit cell. For this large square lattice, the pseudo-Lieb character emerges at bias voltages as low as $-0.30 \mathrm{~V}$ and $-0.15 \mathrm{~V}$ for the bottom and flat bands, respectively. At higher bias voltages, a super-Lieb character appears, as depicted in Fig. 4g. The higher on-site energies of the 'bridging sites' results in a band gap between the lowerenergy bands (which retain their square/Lieb character) and the 
higher-energy bands where localization is more pronounced on the bridging sites.

The ability to generate electronic lattices using $\mathrm{CO}$ molecules on $\mathrm{Cu}(111)$ opens the path to the experimental realization and characterization of many $2 \mathrm{D}$ geometries for which non-trivial properties dictated by the lattice have been anticipated theoretically. Typical examples, which apply to the studied Lieb lattice geometry, are the quantum spin Hall effect, the super-Klein tunnelling paradox, and the Hofstadter butterfly ${ }^{5,11,30,31}$. The $\mathrm{Cu}(111) / \mathrm{CO}$ system is an ideal model system, as it allows one to tune parameters that cannot be easily varied in a real solid-state material. In addition, one can create junctions and study the effects of disorder, which can be designed in a controlled manner. The inherent versatility and the direct access to structural and electronic characterization allow a reality check for advanced theory and a first step in the design of truly novel electronic materials.

\section{Methods}

Methods, including statements of data availability and any associated accession codes and references, are available in the online version of this paper.

Received 14 November 2016; accepted 21 March 2017; published online 24 April 2017

\section{References}

1. Castro Neto, A. H., Guinea, F., Peres, N. M. R., Novoselov, K. S. \& Geim, A. K. The electronic properties of graphene. Rev. Mod. Phys. 81, 109-162 (2009).

2. Weeks, C. \& Franz, M. Topological insulators on the Lieb and perovskite lattices. Phys. Rev. B 82, 085310 (2010).

3. Guo, H. M. \& Franz, M. Topological insulator on the kagome lattice. Phys. Rev. B 80, 113102 (2009).

4. Apaja, V., Hyrkäs, M. \& Manninen, M. Flat bands, Dirac cones, and atom dynamics in an optical lattice. Phys. Rev. A 82, 041402(R) (2010).

5. Goldman, N., Urban, D. F. \& Bercioux, D. Topological phases for fermionic cold atoms on the Lieb lattice. Phys. Rev. A 83, 063601 (2011).

6. Beugeling, W., Everts, J. C. \& Smith, C. M. Topological phase transitions driven by next-nearest-neighbor hopping in two-dimensional lattices. Phys. Rev. B 86, 195129 (2012).

7. Tadjine, A., Allan, G. \& Delerue, C. From lattice Hamiltonians to tunable electron band structures by lithographic design. Phys. Rev. B 94, 075441 (2016)

8. Van Miert, G. \& Smith, C. M. Dirac cones beyond the honeycomb lattice: a symmetry-based approach. Phys. Rev. B 93, 035401 (2016).

9. Li, S., Qiu, W.-X. \& Gao, J.-H. Designing artificial two dimensional electron lattice on metal surface: a Kagome-like lattice as an example. Nanoscale $\mathbf{8}$, 12747-12754 (2016).

10. Di Liberto, M., Hemmerich, A. \& Smith, C. M. Topological Varma superfluid in optical lattices. Phys. Rev. Lett. 117, 163001 (2016).

11. Shen, R., Shao, L. B., Wang, B. \& Xing, D. Y. Single Dirac cone with a flat band touching on line-centered-square optical lattices. Phys. Rev. B 81, 041410(R) (2010).

12. Guzmán-Silva, D. et al. Experimental observation of bulk and edge transport in photonic Lieb lattices. New J. Phys. 16, 063061 (2014).

13. Mukherjee, S. et al. Observation of a localized flat-band state in a photonic Lieb lattice. Phys. Rev. Lett. 114, 245504 (2015).

14. Vicencio, R. A. et al. Observation of localized states in Lieb photonic lattices. Phys. Rev. Lett. 114, 245503 (2015).

15. Taie, S. et al. Coherent driving and freezing of bosonic matter wave in an optical Lieb lattice. Science Adv. 1, 1500854 (2015).
16. Xia, S. et al. Demonstration of flat-band image transmission in optically induced Lieb photonic lattices. Opt. Lett. 41, 1435-1438 (2016).

17. Diebel, F., Leykam, D., Kroesen, S., Denz, C. \& Desyatnikov, A. S. Conical diffraction and composite Lieb bosons in photonic lattices. Phys. Rev. Lett. 116, 183902 (2016)

18. Lieb, E. H. Two theorems on the Hubbard model. Phys. Rev. Lett. 62, 1201-1204 (1989).

19. Costa, N. C., Mendes-Santos, T., Paiva, T., Dos Santos, R. R. \& Scalettar, R. T. Ferromagnetism beyond Lieb's theorem. Phys. Rev. B 94, 155107 (2016).

20. Zhao, A. \& Shen, S.-Q. Quantum anomalous Hall effect in a flat band ferromagnet. Phys. Rev. B 85, 085209 (2012).

21. Jaworowski, B., Manolescu, A. \& Potasz, P. Fractional Chern insulator phase at the transition between checkerboard and Lieb lattices. Phys. Rev. B 92, 245119 (2015)

22. Kopnin, N. B., Heikkilä, T. T. \& Volovik, G. E. High-temperature surface superconductivity in topological flat-band systems. Phys. Rev. B 83, 220503(R) (2011)

23. Julku, A., Peotta, S., Vanhala, T. I., Kim, D. H. \& Törmäi, P. Geometric origin of superfluidity in the Lieb-lattice flat band. Phys. Rev. Lett. 117, 045303 (2016)

24. Park, C.-H. \& Louie, S. G. Making massless Dirac fermions from a patterned two-dimensional electron gas. Nano Lett. 9, 1793-1797 (2009).

25. Gomes, K. K., Mar, W., Ko, W., Guinea, F. \& Manoharan, H. C. Designer Dirac fermions and topological phases in molecular graphene. Nature 483, 306-310 (2012).

26. Moon, C. R. et al. Quantum phase extraction in isospectral electronic nanostructures. Science 319, $782-787$ (2008).

27. Moon, C., Mattos, L. S., Foster, B. K., Zeltzer, G. \& Manoharan, H. C. Quantum holographic encoding in a two-dimensional electron gas. Nat. Nanotech. 4, 167-172 (2009)

28. Paavilainen, S., Ropo, M., Nieminen, J., Akola, J. \& Räsänen, E. Coexisting honeycomb and Kagome characteristics in the electronic band structure of molecular graphene. Nano Lett. 16, 3519-3523 (2016).

29. Qiu, W.-X., Li, S., Gao, J.-H., Zhou, Y. \& Zhang, F.-C. Designing an artificial Lieb lattice on a metal surface. Phys. Rev. B 94, 241409 (2016).

30. Nita, M., Ostahie, B. \& Aldea, A. Spectral and transport properties of the two-dimensional Lieb lattice. Phys. Rev. B 87, 125428 (2013).

31. Urban, D. F., Bercioux, D. \& Wimmer, M. Barrier transmission of Dirac-like pseudospin-one particles. Phys. Rev. B 84, 115136 (2011).

\section{Acknowledgements}

Financial support from the Foundation for Fundamental Research on Matter (FOM, grants 16PR3245 and DDC13), which is part of the Netherlands Organisation for Scientific Research (NWO), as well as the European Research Council ('FIRSTSTEP',692691) is gratefully acknowledged. We thank J. van der Lit and N. van der Heijden for fruitful discussions.

\section{Author contributions}

M.R.S., T.S.G., P.H.J. and I.S. planned the experiment, including the proposal of the design of the CO lattice. M.R.S. and T.S.G. performed the experiments and analysed the data. P.H.J. carried out the tight-binding calculations and G.C.P.v.M. performed the muffin-tin model calculations. S.J.M.Z. developed a program that partially automates the lattice assembly. All authors contributed to the discussions and the manuscript.

\section{Additional information}

Supplementary information is available in the online version of the paper. Reprints and permissions information is available online at www.nature.com/reprints. Publisher's note: Springer Nature remains neutral with regard to jurisdictional claims in published maps and institutional affiliations. Correspondence and requests for materials should be addressed to I.S.

\section{Competing financial interests}

The authors declare no competing financial interests. 


\section{Methods}

Scanning tunnelling microscope (STM) experiments. The experiments were performed in a Scienta Omicron LT-STM, operating at a temperature of $4.6 \mathrm{~K}$ and a pressure in the $10^{-10} \mathrm{mbar}$ range. Prior to the experiments, a clean $\mathrm{Cu}(111)$ crystal surface was prepared by several cycles of sputtering and annealing. After cooling down in the STM microscope head, CO was deposited on the surface by leaking in this gas to $P=2 \times 10^{-8} \mathrm{mbar}$ for $3 \mathrm{~min}$. For all measurements a $\mathrm{Cu}$-coated tungsten tip was used. Assisted by an in-house developed program, atomic manipulations were performed following previously described procedures ${ }^{32,33}$. STM images were acquired in constant current mode. $\mathrm{d} I / \mathrm{d} V$ spectroscopy and mapping were performed in constant-height mode using a standard lock-in amplifier modulating the sample bias with an amplitude of $10-20 \mathrm{mV}$ r.m.s. at a frequency of $273 \mathrm{~Hz}$. Various tips, characterized by differently shaped $\mathrm{Cu}(111)$ spectra, were used to corroborate the features arising from the Lieb lattice.

Tight-binding calculations. Tight-binding calculations were performed for periodic and finite-sized lattices. For dispersion and LDOS calculations, we utilized a grid of $50 \times 50 \mathrm{k}$-points in the first Brillouin zone, whereas $n \times n \mathrm{k}$-points were used for calculating the differential conductance maps of the higher-order lattices. The used tight-binding parameters were $t^{\prime} / t=0.6$ and an orbital overlap of $s=0.15$ The calculations on the experimentally realized geometry are $\Gamma$-point calculations with periodic boundary conditions, utilizing the same tight-binding parameters as the periodic lattice calculations. The local density of states was inferred directly from sites in the centre of both lattices, using a Lorentzian energy level broadening of $\Gamma=0.8 t$. Simulated differential conductance maps were obtained by taking again $\Gamma=0.8 t$ and by expanding the wave functions by normalized Gaussians of width $\sigma=0.4 a$, where $a$ is the lattice constant of the Lieb lattice.

Muffin-tin calculations. The surface state electrons of $\mathrm{Cu}(111)$ can be considered a $2 \mathrm{D}$ electron gas. The $\mathrm{CO}$ molecules are modelled as disks (radius $0.3 \mathrm{~nm}$ ), centred at a CO molecule, with a repulsive potential of $0.9 \mathrm{eV}$. See Supplementary Information for details.

Data availability. The data that support the plots within this paper and other findings of this study are available from the corresponding author upon reasonable request.

\section{References}

32. Meyer, G. et al. Controlled manipulation of atoms and small molecules with a low temperature scanning tunneling microscope. Single Mol. 1, 79-86 (2000).

33. Celotta, R. J. et al. Invited article: autonomous assembly of atomically perfect nanostructures using a scanning tunneling microscope. Rev. Sci. Instrum. 85, $121301(2014)$ 